\title{
Radiation-induced abscopal effect and its enhancement by programmed cell death 1 blockade in the hepatocel- lular carcinoma: A murine model study
}

Gyu Sang Yoo ${ }^{1,2, *}$, Won-Gyun Ahn ${ }^{1, *}$, Shin-Yeong Kim ${ }^{1}$, Wonseok Kang ${ }^{2,3}$, Changhoon Choi', and Hee Chul Park ${ }^{1,2}$

'Department of Radiation Oncology, Samsung Medical Center, Seoul; ${ }^{2}$ Sungkyunkwan University School of Medicine, Seoul; ${ }^{3}$ Department of Medicine, Samsung Medical Center, Seoul, Korea

Graphical Abstract

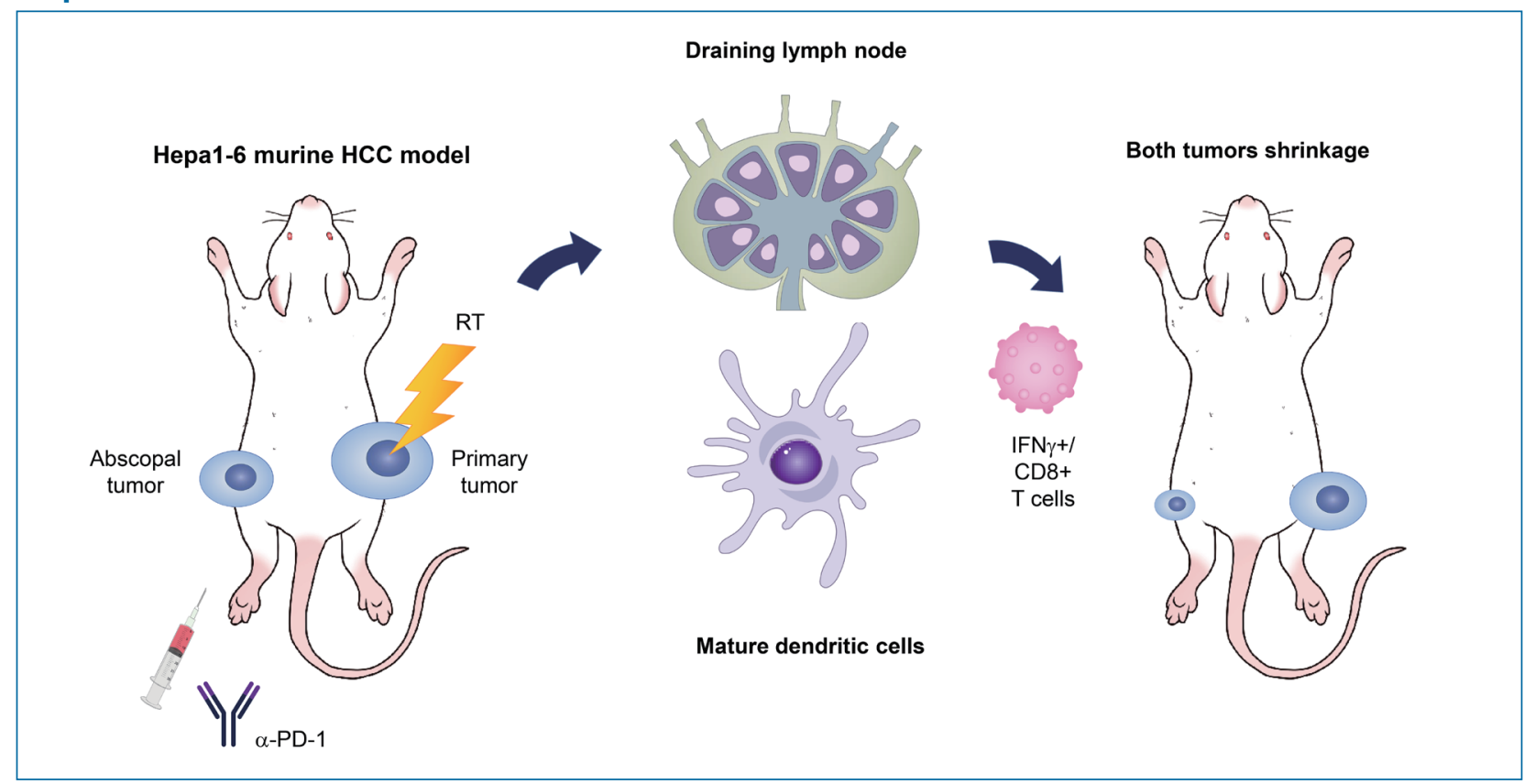

\section{Abbreviations:}

cGAS, cyclic guanosine monophosphate-adenosine monophosphate synthase CTLA-4, cytotoxic T lymphocyte-associated protein-4; DC, dendritic cell; HCC, hepatocellular carcinoma; ICI, immune checkpoint inhibitor; IFN, interferon; MDSCs, myeloid-derived suppressor cells; ORRs, objective response rates; PD-1, programmed cell death 1; PD-L1, programmed cell death ligand 1; RT, radiation therapy; STING, stimulator of interferon gene; TDLNs, tumor-draining lymph nodes
Corresponding author : Changhoon Choi

Department of Radiation Oncology, Samsung Medical Center, 81 Irwonro, Gangnam-gu, Seoul 06351, Korea

Tel: +82-2-6190-5331, Fax: +82-2-3410-2619

E-mail:chchoi93@gmail.com

https://orcid.org/0000-0002-2847-1168

\section{Hee Chul Park}

Department of Radiation Oncology, Samsung Medical Center, 81 Irwonro, Gangnam-gu, Seoul 06351, Korea

Tel: +82-2-3410-2612, Fax: +82-2-3410-2619

E-mail: hee.ro.park@samsung.com

https://orcid.org/0000-0003-0385-9681

*These authors contributed equally to this work. 
Background/Aims: The abscopal effect, a rare phenomenon induced by radiation, can be reinforced by immunotherapy. Although radiation therapy and immunotherapy are increasingly being utilized for the treatment of hepatocellular carcinoma (HCC), whether immunotherapy could boost the abscopal effect remains unclear. In this study, we aimed to elucidate the immunological mechanisms underlying the abscopal effect induced by the combination of irradiation and immunotherapy in a murine HCC model.

Methods: A syngeneic HCC mouse model was established by transplanting murine Hepa 1-6 HCC cells into both hind legs of immunocompetent C57BL/6 mice. The tumors on the right hind legs were irradiated, and abscopal effects were observed in the non-irradiated tumors on the left hind leg with or without the coadministration of anti-programmed cell death 1 (PD-1) antibodies. Flow cytometric analyses were performed to analyze the distributions of immune cells infiltrating both irradiated and non-irradiated tumors and the tumor-draining lymph nodes (TDLNs).

Results: Administration of $16 \mathrm{~Gy}$ in two fractions more effectively inhibited the growth of both irradiated and nonirradiated tumors with higher tumor infiltration of cytotoxic T cells than $8 \mathrm{~Gy}$ did in a single fraction. The higher dose also increased activated dendritic cells in TDLNs, which had higher expression of the programmed cell death ligand 1. Coadministration of anti-PD-1 antibodies significantly enhanced the abscopal effect and increased infiltration of activated cytotoxic $T$ cells in both irradiated and non-irradiated tumors.

Conclusions: Our findings show that adding anti-PD-1 therapy to radiation enhanced the abscopal effect in a syngeneic murine model of HCC. (Clin Mol Hepatol 2021;27:144-156)

Keywords: Immunotherapy; Radiotherapy; Carcinoma, Hepatocellular; Murine model

\section{Study Highlights}

In this study, a syngeneic HCC mouse model was established by transplanting murine Hepa 1-6 HCC cells into both hind legs of immunocompetent C57BL/6 mice. The tumors on the right hind legs were irradiated, and abscopal effects were observed in the non-irradiated tumors on the left hind leg with or without the coadministration of anti-PD-1 antibodies. We observed that the coadministration of anti-PD-1 antibodies significantly enhanced the abscopal effect and increased infiltration of activated cytotoxic T cells in both irradiated and non-irradiated tumors.

\section{INTRODUCTION}

The abscopal effect, which refers to the regression of tumors outside the radiation field during or after radiation therapy (RT), has been reported in various cancers such as melanoma, renal cell carcinoma, and breast cancer for over 60 years. ${ }^{1}$ Although its mechanism has not yet been fully elucidated, the abscopal effect is likely mediated by the radiation-induced enhancement of systemic immune responses. ${ }^{2}$ However, the abscopal effect has been rarely observed, possibly because of the immune escape mechanisms of cancer cells, including the activation of the immune checkpoint pathway. ${ }^{3}$ Newly developed immune checkpoint inhibitors (ICIS) have revolutionized cancer treatment over the last decade. ${ }^{4}$ Following the emergence of ICls that enhance systemic immune responses against cancer cells, new therapeutic regimens combining ICls and RT are expected to boost the abscopal effect. ${ }^{5}$ The synergistic effects of ICls and RT are being actively investigated and the potential of this combination to intensify the abscopal effect has been reviewed in various reports. ${ }^{6-8}$

Hepatocellular carcinoma (HCC) is the most common type of primary liver cancer and the second leading cause of cancer-related death worldwide. ${ }^{9,10}$ Patients in the early stage of HCC treated with curative modalities such as resection, liver transplantation, and ablation show an overall survival rate $>60 \%$, whereas those with advanced HCC who undergo chemoembolization or systemic therapy have a dismal prognosis. ${ }^{11,12}$ To improve this poor outcome, new regimens have recently been incorporated and suggested in the treatment of $\mathrm{HCC}$, including considering the use of ICls. Therefore, the feasibility and safety of various ICls, including programmed cell death 1 (PD-1) inhibitors, PD ligand 1 (PD-L1) inhibitors, and cytotoxic T lymphocyte-associated protein-4 (CTLA4) inhibitors, are under investigation in patients with $\mathrm{HCC}$. ${ }^{13-17}$ However, the reported objective response rates (ORRs) of ICls were only $15-20 \%$, which are still unsatisfactory. ${ }^{14-16}$ Furthermore, the recent KEYNOTE-240 trial evaluating the efficacy and safety of pembrolizumab in patients with advanced HCC failed to show 
statistical significance in the improvement of overall survival and progression-free survival compared with the placebo. ${ }^{18}$ RT has received considerable attention for use in enhancing the efficacy of ICls for advanced HCC because of the abscopal phenomenon induced by its immunogenicity. ${ }^{19}$

The efficacy of cotreatment in controlling primary HCC has been recently tested in a few preclinical experiments and clinical trials. ${ }^{20-22}$ However, these previous studies did not use metastatic $\mathrm{HCC}$ models but rather used orthotopic models, which only enable the observation of the combined effect of RT and ICls on primary tumors but not the abscopal effect. Therefore, in this study, we aimed to investigate the effect and immunological mechanisms of action of radiation on the abscopal phenomenon and the effect of an anti-PD-1 antibody on the abscopal effect using a bilateral murine syngeneic $\mathrm{HCC}$ model.

\section{MATERIALS AND METHODS}

\section{Cell culture}

The murine Hepa 1-6 HCC cell line was purchased from the American Type Culture Collection (ATCC, Manassas, VA, USA). Hepa 1-6 cells were cultured in Dulbecco's modified Eagle's medium (DMEM; Gibco, Carlsbad, CA, USA) containing 10\% fetal bovine serum and $1 \%$ antibiotic-antimycotic solution (Thermo Fisher Scientific, Waltham, MA, USA) at $37^{\circ} \mathrm{C}$ exposed to an atmosphere of $5 \% \mathrm{CO}_{2}$.

\section{Animal model}

Male C57BL/6 mice (5-6 weeks old) were purchased from Orient Bio (Seongnam, Korea). To establish the syngeneic mouse model of bilateral HCC tumors, Hepa $1-6$ cells $\left(1 \times 10^{6}\right.$ cells/ mouse) were injected into the right hind leg and then cells were also injected into the left hind leg 3 days after the first injection. Tumor dimensions were measured every 3 days using calipers and the volume was calculated according to the following formula: volume $=\mathrm{W}^{2} \times \mathrm{L} / 2$ (L, length [mm]; W, width [mm]).

When the mean tumor volume reached $300 \mathrm{~mm}^{3}$, the mice were randomized into various treatment groups ( $n \geq 5$ per group) and after 30 days they were euthanized, followed by tumor collection for further analysis. All animal procedures were conducted in accordance with appropriate regulatory standards under the study protocol (ID: 20181227001, approval date: 2019-03-08), which was reviewed and approved by the Institutional Animal Care and Use Committee of Samsung Biomedical Research Institute.

\section{Drug and radiation treatment}

For irradiation, $6 \mathrm{MV}$ photon beams were delivered to the tumor-bearing right hind leg at a dose rate of $3.96 \mathrm{~Gy} / \mathrm{min}$ using a linear accelerator (Varian Medical System, Palo Alto, CA, USA) as described previously. ${ }^{23,24}$ Irradiation was performed at a single 8 Gy or total 16 Gy dose in two fractions. Before irradiation, the mice were anesthetized via intraperitoneal injections of $30 \mathrm{mg} / \mathrm{kg}$ zolazepam/tiletamine and $10 \mathrm{mg} / \mathrm{kg}$ xylazine. All mice were placed in a prone position and the tumor-bearing right hind legs were placed under a 2-cm-thick water-equivalent bolus with a source-to-surface distance of $100 \mathrm{~cm}$ and radiation field size of $30 \times 7 \mathrm{~cm}$. The mice were positioned to minimize the radiation dose administered to the tumor-draining inguinal lymph nodes. Details on the experimental setup are provided in Supplementary Figure 1. Starting on day 14 , the mouse anti-PD-1 antibodies and isotype control (BE0146 and BE0089, respectively; Bio X cell, West Lebanon, NH, USA) were administered intraperitoneally once a week at a dose of $2 \mathrm{mg} / \mathrm{kg}$.

\section{Flow cytometry analysis}

On day 31 after the first injection of Hepa 1-6 cells, tumors were harvested, a single cell suspension was prepared for flow cytometry analysis, and red blood cells were removed by incubation with Pharm Lyse (BD Biosciences, San Jose, CA, USA) for 30 minutes at $4^{\circ} \mathrm{C}$. The cells were fixed with Cytofix (BD Biosciences) for 30 minutes at $4^{\circ} \mathrm{C}$ and then they were resuspended in staining buffer (BD Biosciences). For T cell analysis, cells were permeabilized using the Fix/Perm buffer kit (eBioscience, San Diego, CA, USA) and then they were stained with anti-forkhead box P3 (Foxp3, BD560408) and anti-interferon (IFN)- (BD557724) antibodies for 30 minutes at $4^{\circ} \mathrm{C}$. After washing, cells were stained with antibodies specific for CD4 (BD55205), CD8 (BD560469), CD3e (BD560771), and CD25 (BD551071).

For dendritic cell (DC) analysis, inguinal lymph nodes of the irradiated tumor side were collected 3 days after irradiation, and DCs were isolated using the EasySep ${ }^{\mathrm{TM}}$ mouse plasmacytoid DC isolation kit (\#19764, STEMCELL Technologies, Vancouver, Canada). Furthermore, $1 \times 10^{5}$ DCs were stained with CD11C (BD553801), CD40 (BD562846), CD80 (BD560016), and PD-L1 (12-5982, eBioscience; Thermo Fisher Scientific). Data were acquired using a flu- 
orescence activated cell sorting verse flow cytometer (BD Biosciences) and were analyzed using FlowJo software (Tree Star Inc., Ashland, OR, USA).

\section{Statistical analysis}

All data are presented as the means \pm standard deviation. Statistical analysis was performed using a one-way analysis of variance with Bonferroni's correction using Prism 8.0 (GraphPad Software, San Diego, CA, USA). A $P<0.05$ was considered statistically significant and survival curves were assessed using the Kaplan-Meier analysis with a log-rank test. $P$ values are indicated in the figures as follows: ${ }^{*} P<0.05,{ }^{\dagger} P<0.01$, and ${ }^{\ddagger} P<0.001$.

\section{RESULTS}

\section{Localized tumor irradiation evokes the abscopal response in a syngeneic HCC mouse model}

A syngeneic bilateral HCC mouse model was established using Hepa 1-6 cells (Fig. 1A). The tumor growth curves showed that irradiation with $16 \mathrm{~Gy}$ in two fractions but not $8 \mathrm{~Gy}$ in a single fraction more significantly inhibited tumor growth $(P<0.001$, Fig. $1 \mathrm{~B})$ and non-irradiated tumors $(P<0.05$, Fig. $1 \mathrm{C})$ than the sham control, indicating that the regimen of 16 Gy in two fractions was applicable for evoking abscopal effects in the present HCC model. Tumors harvested on day 31 were imaged (Fig. 1D), and irradiation with 16 Gy in two fractions significantly improved the survival of mice more than the sham control treatment or irradiation with a single fraction of $8 \mathrm{~Gy}$ did $(P<0.001$, Fig. 1E).

\section{Localized tumor irradiation induces infiltration of activated T cells in both irradiated and non- irradiated tumors}

To determine whether the reduction of non-irradiated tumor size by localized irradiation may be associated with immunogenic activation, we performed flow cytometry analysis of tumor infiltrating lymphocytes (Fig. 2A, B). Quantification of the data showed that 16 Gy administered in two fractions but not 8 Gy in a single fraction significantly increased infiltration of $\mathrm{CD}^{+} \mathrm{IFN}-\gamma^{+}$ and $\mathrm{CD}^{+} \mathrm{IFN}-\gamma^{+} \mathrm{T}$ cells in both irradiated $(P<0.001$ and $P<0.05$, Fig. 2C) and non-irradiated tumors $(P<0.001$ and $P<0.001$, Fig. 2D). Furthermore, 16 Gy administered in two fractions also in- creased infiltration of regulatory T cells (Treg, $C D 4^{+} \mathrm{CD} 25^{+}$Foxp $^{+}$) in irradiated tumors $(P<0.05$, Fig. $2 C)$.

\section{Localized tumor irradiation activates DCs in tumor- draining lymph nodes (TDLNs)}

To understand how localized irradiation reduces non-irradiated tumors, we examined DCs in the inguinal lymph nodes draining from tumors implanted in the right hind legs of the mice using flow cytometry (Fig. 3A, B). Quantification data show that 16 Gy administered in two fractions significantly increased the frequency of activated DCs in the TDLNs, as evidenced by the high expression of CD40 $(P<0.001)$ and CD80 $(P<0.01$, Fig. 3C). Expression of PD-L1, the co-inhibitory molecule on CD11 $\mathrm{C}^{+} \mathrm{DC}$ in the TDLNs, was also induced by irradiation with two fractions of $8 \mathrm{~Gy}$, compared to the sham treatment $(P<0.05$, Fig. 3C). Moreover, $16 \mathrm{~Gy}$ administered in two fractions also activated T cells in the TDLNs, as evidenced by a significant increase in $C D 4^{+} I F N-\gamma^{+}$and CD8 ${ }^{+}$IFN $-\gamma^{+} T$ cells $(P<0.001$, Fig. 3D). Accumulation of Tregs in the TDLNs was also more evident in the group administered two 8 Gy fractions than in the sham-treated group or the group administered a single 8 Gy fraction (Fig. 3D).

\section{Cotreatment with radiation and anti-PD-1 antibodies boosts the abscopal effect in the syngeneic HCC mouse model}

Based on the result showing that radiation increased the expression of PD-L1 in the TDLN-resident DCs, we determined whether blockade of the PD-1/PD-L1 pathway could further enhance the abscopal effect in the syngeneic, bilateral HCC mouse model (Fig. 4A). Anti-PD-1 antibodies inhibited the growth of bilateral Hepa 1-6 tumors more than the isotype IgG control did (Fig. 4C), but the difference was not statistically significant.

As expected, $16 \mathrm{~Gy}$ administered in two fractions inhibited the growth of both irradiated $(P<0.001$, Fig $4 \mathrm{~B})$ and non-irradiated abscopal tumors (Fig. 4C, not significant). Cotreatment with radiation and anti-PD-1 further suppressed the growth of both irradiated and non-irradiated tumors more than radiation alone or antiPD-1 monotherapy did (Fig. 4B, C). Tumors harvested from mice cotreated with the anti-PD-1 antibody and radiation were clearly smaller than those from mice treated with radiation or anti-PD-1 monotherapy (Fig. 4D). Anti-PD-1 treatment alone did not improve survival but in combination with radiation, it increased survival significantly $(P<0.001$, Fig. $4 \mathrm{E})$. 


\section{Combination of radiation and anti-PD-1 antibodies further enhances T lymphocyte infiltration of abscopal HCC tumors}

Immunohistochemistry using antibodies specific to CD4 and CD8 showed that anti-PD-1 antibodies and radiation alone more significantly increased the infiltration of $\mathrm{CD}^{+}$and $\mathrm{CD} 8^{+}$cells into irradiated tumors than the IgG control did $(P<0.05$; Supplementary Fig. $2 A, B)$, along with $C D 4^{+}$cells into the non-irradiated contralateral tumors $(P<0.01$ and $P<0.001$; Supplementary Fig. $2 C$, D). Cotreatment with the anti-PD-1 antibodies and radiation further enhanced infiltration of $\mathrm{CD}^{+}$cells into both tumors, although no additive effect was obvious.

Flow cytometry analysis of tumors harvested on day 31 (Fig. 5A)

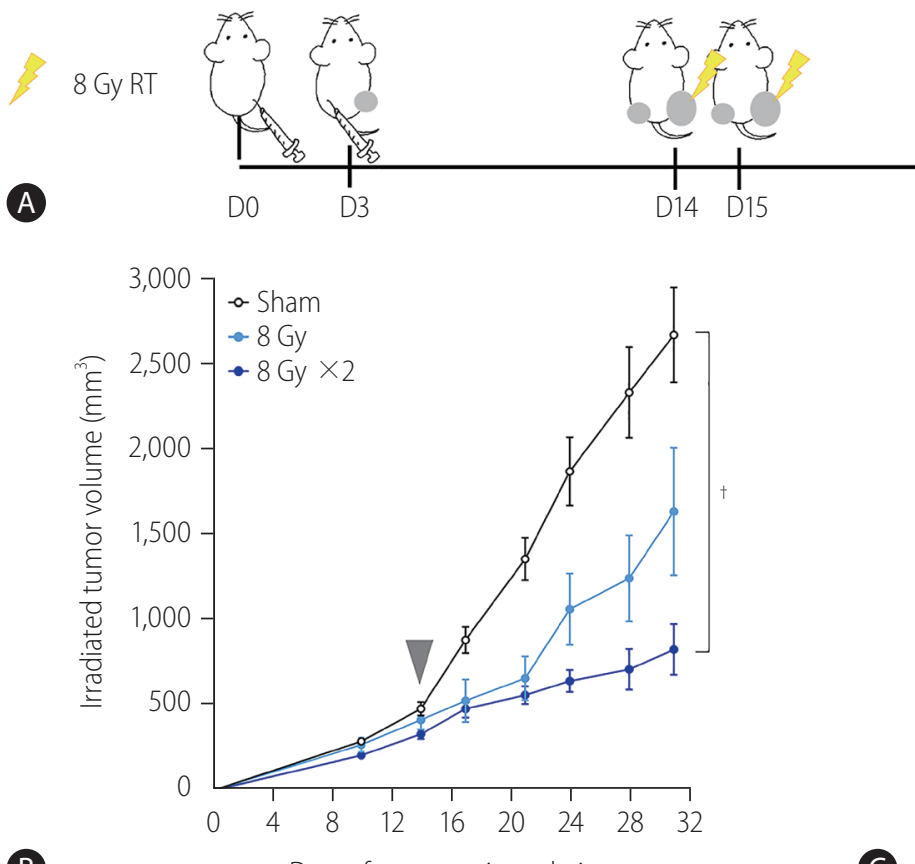

B Days after tumor inoculation

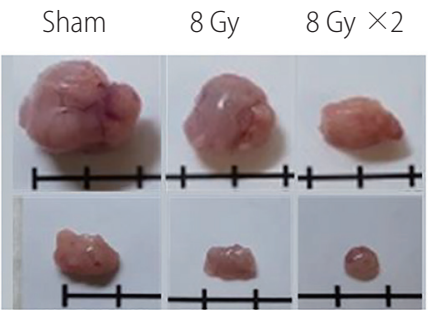

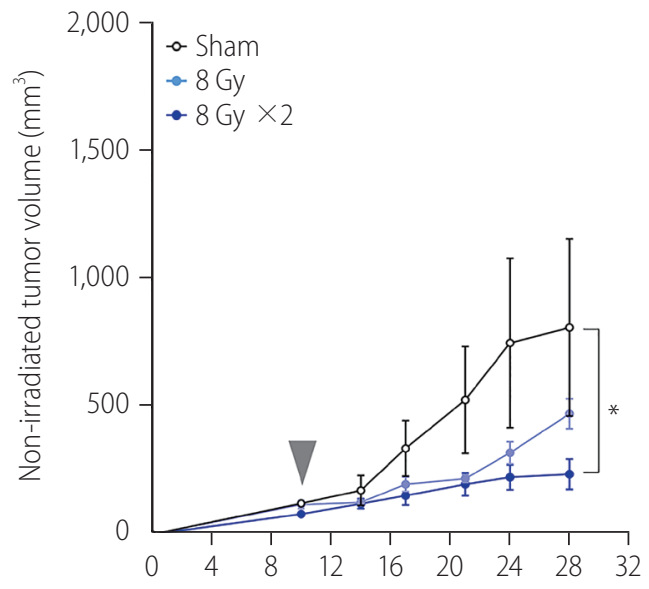

C

Days after tumor inoculation

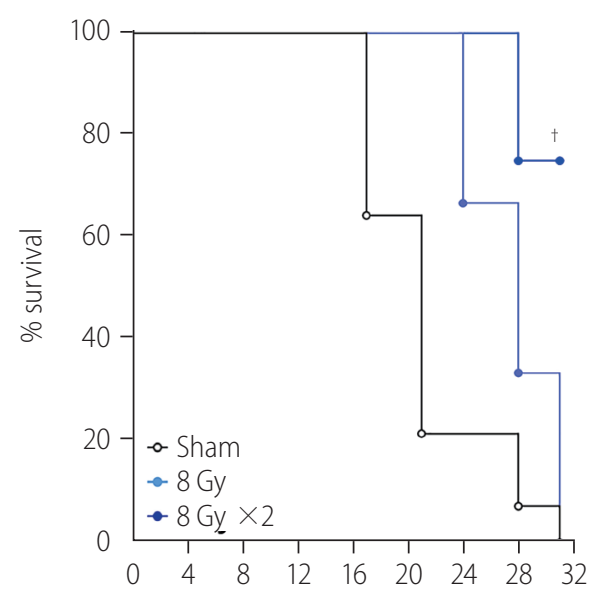

E

Days after tumor inoculation

Figure 1. Localized irradiation inhibits the growth of both primary and distant tumors in a syngeneic model of murine hepatocellular carcinoma. (A) Scheme for radiation treatment of a bilateral tumor mouse model established using Hepa 1-6 cells. Growth curves of (B) irradiated tumors and (C) non-irradiated tumors in mice bearing bilateral Hepa 1-6 tumors. Gray arrowheads indicate day 1 of irradiation. (D) Representative images of tumors collected from mice. (E) Survival of mice bearing bilateral Hepa 1-6 tumors for three different treatment groups. RT, radiation therapy. ${ }^{*} P<0.05$. ${ }^{\dagger} P<0.001$. 

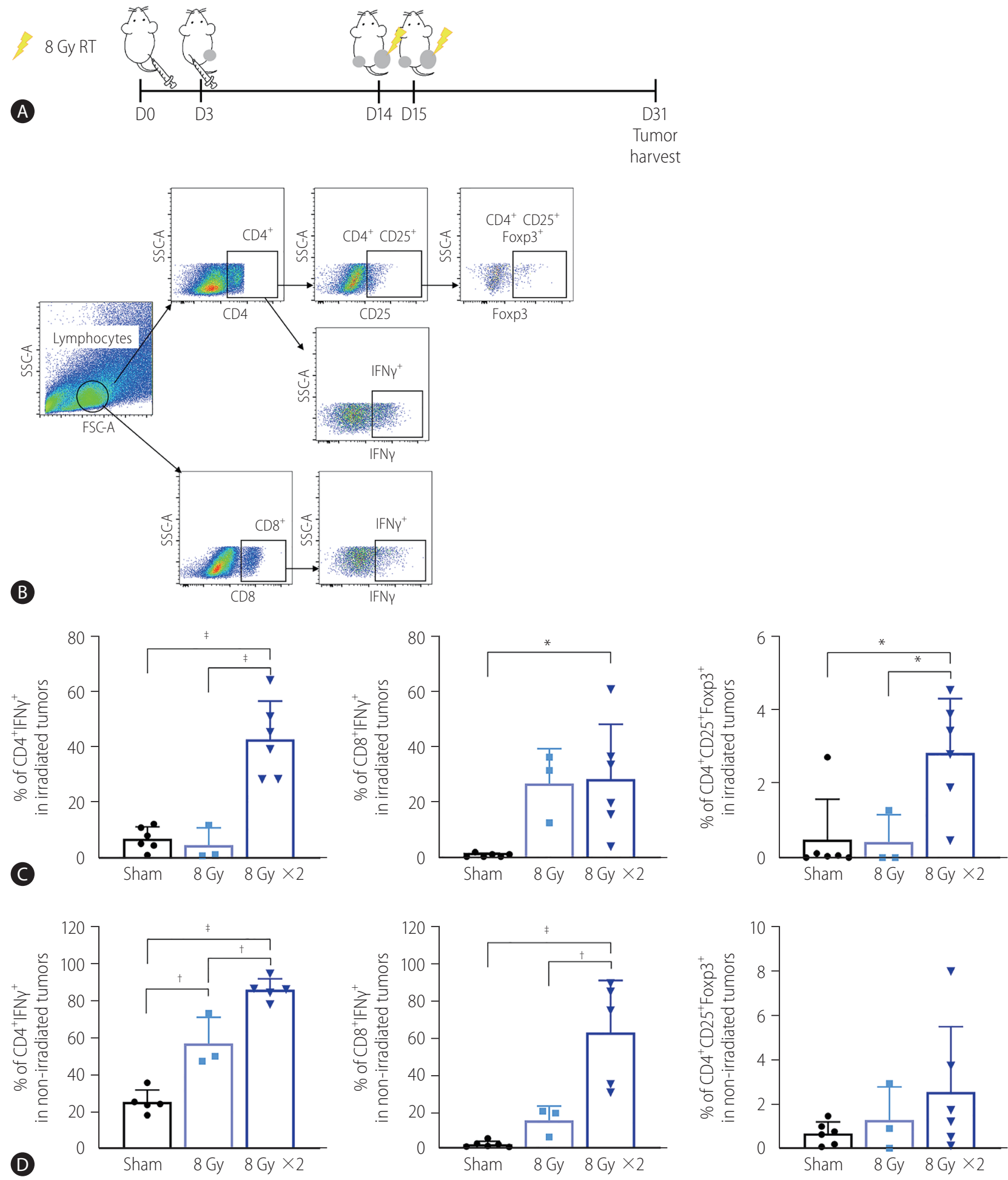

Figure 2. Augmented radiation doses increased infiltration of activated T cells into non-irradiated and irradiated tumors. (A) Scheme for radiation treatment of a bilateral tumor mouse model established using Hepa 1-6 cells. (B) Representative flow cytometry dot plots showing profiles of activated T cell populations and regulatory T cells (Tregs). (C) Quantification data showed that administration of two but not one 8 Gy fraction significantly increased the population of IFNy expressing $\mathrm{CD}^{+}$and $\mathrm{CD}^{+} \mathrm{T}$ cells and Tregs in the irradiated tumors. (D) Two fractions of 8 Gy also significantly increased the population of $\mathrm{CD}^{+}$and $\mathrm{CD} 8^{+} \mathrm{T}$ cells expressing IFNy cells in non-irradiated tumors. RT, radiation therapy; SSC-A, side scatter analysis; FSC-A, forward scatter analysis; IFN, interferon. ${ }^{*} P<0.05 .{ }^{\dagger} P<0.01 .{ }^{\ddagger} P<0.001$. 


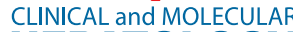

\section{HEPATOLOGY}

Volume_27 Number_1 January 2021
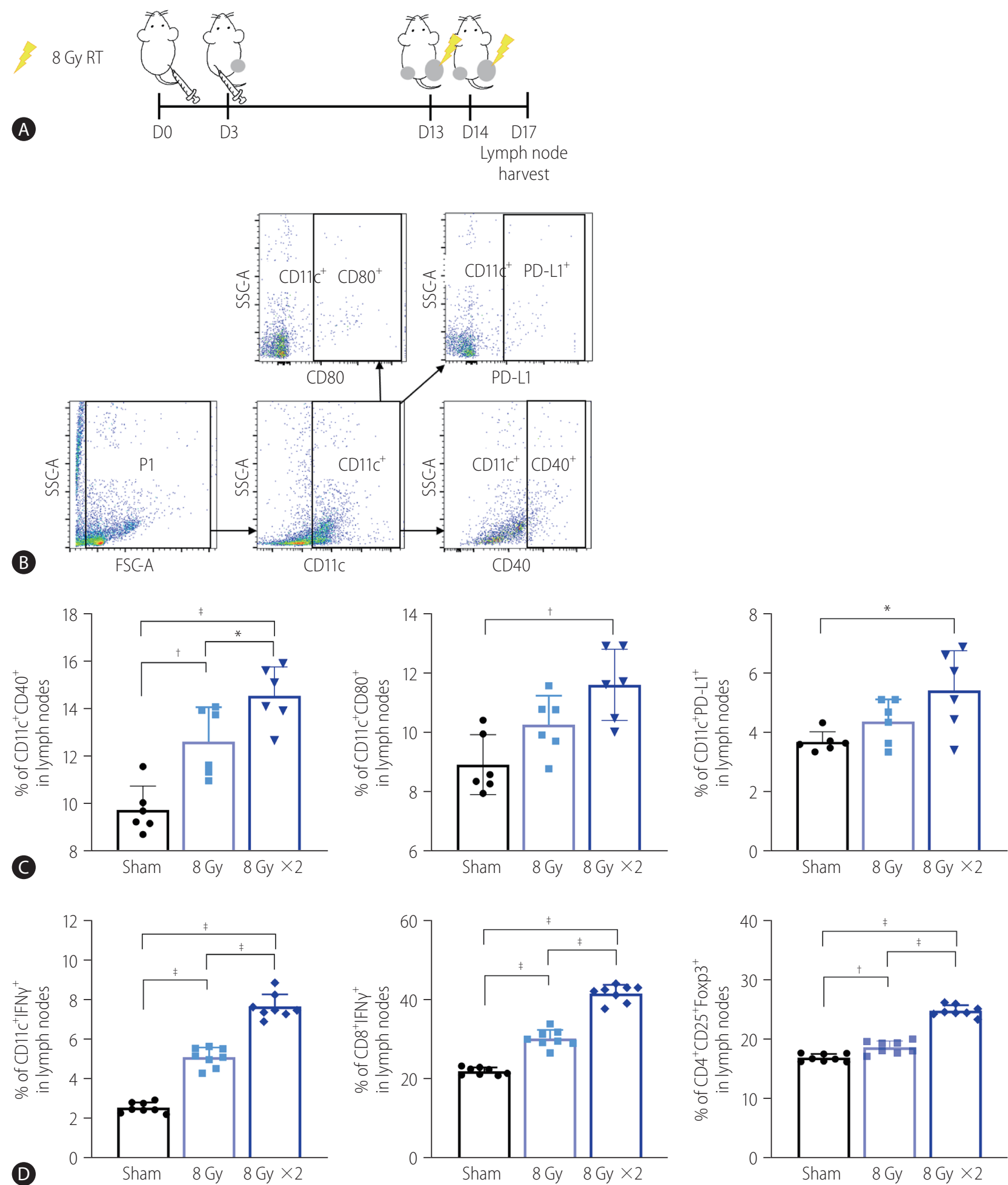

Figure 3. Irradiation of tumor sites increased activation of DCs and T lymphocytes in TDLNs. (A) Scheme for radiation treatment of a bilateral Hepa 1-6 tumor model. (B) Representative flow cytometry dot plots showing profiles of activated DC populations and PD-L1 expressing DCs. (C) Quantification data showed that two fractions of 8 Gy radiation increased activated populations of CD11 ${ }^{+}$DCs in TDLNs, as evidenced by CD40 and CD80 expression. Radiation also increased PD-L1 expression of CD11 ${ }^{+}$DCs. (D) Both single and two fractions of 8 Gy increased activated T cell populations in TDLNs. RT, radiation therapy; PD-L1, programmed cell death 1 ligand 1; SSC-A, side scatter analysis; FSC-A, forward scatter analysis; IFN, interferon; DCS, dendritic cells; TDLNs, tumor-draining lymph nodes. ${ }^{*} P<0.05 .{ }^{\dagger} P<0.01$. ${ }^{\ddagger} P<0.001$. 
8 Gy RT

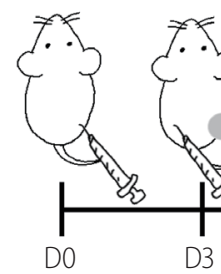

A $40 \mu \mathrm{g}$ aPD-1

or isotype $\lg G$

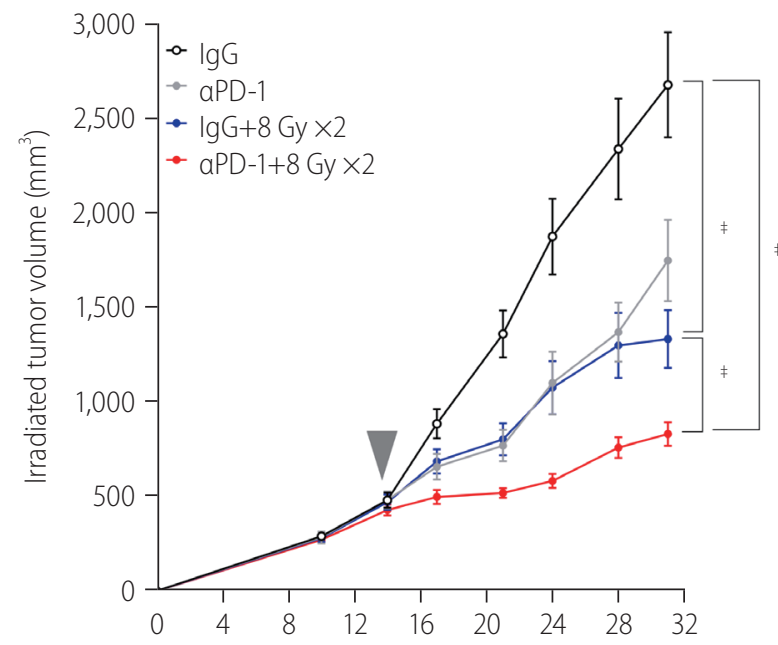

B

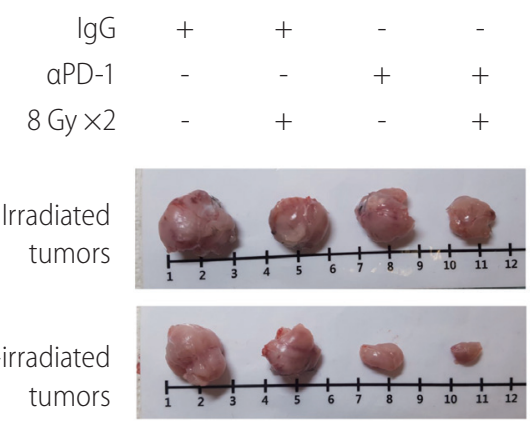

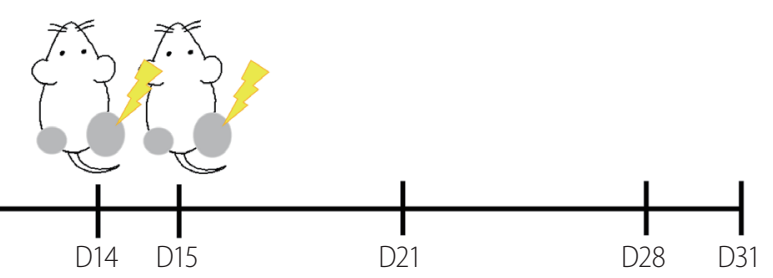

$\mathbb{P}$

Y"

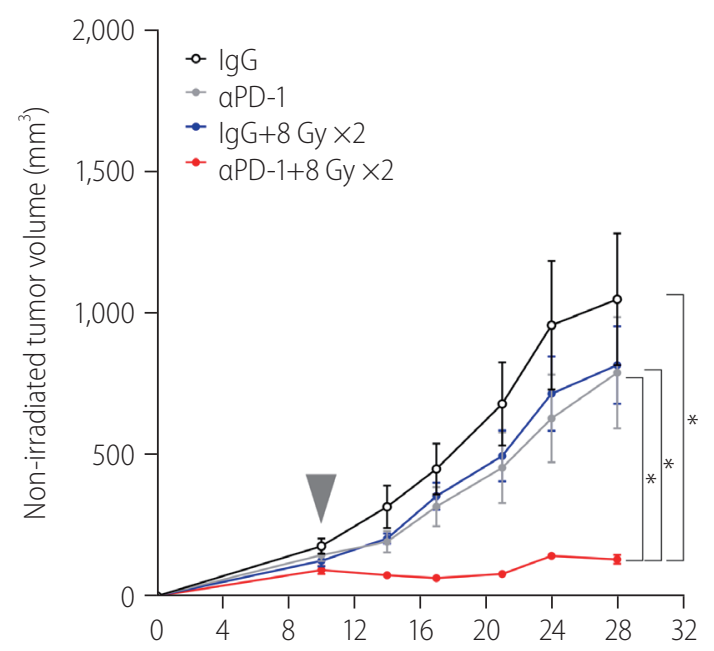

C

Days after tumor inoculation

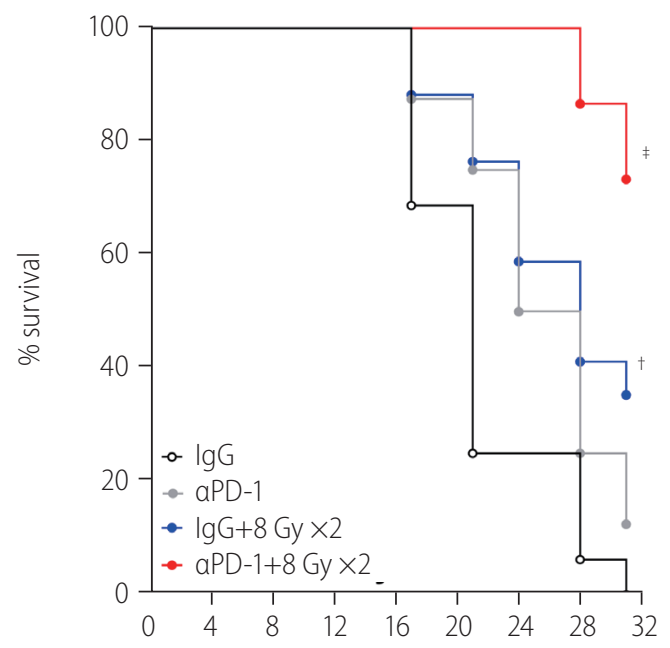

E

Days after tumor inoculation

Figure 4. Administration of anti-PD-1 enhances the antitumor effect of radiation on both irradiated and non-irradiated tumors. (A) Scheme for antiPD-1 and radiation treatment of a bilateral Hepa 1-6 tumor mouse model. Growth curves of (B) irradiated and (C) non-irradiated tumors in mice bearing bilateral Hepa 1-6 tumors. Gray arrowheads indicate day 1 of irradiation. (D) Representative images of tumors collected from mice. (E) Survival of mice bearing bilateral Hepa 1-6 tumors from four different treatment groups. RT, radiation therapy; PD-1, programmed cell death 1; lgG, immunoglobulin G. ${ }^{*} P<0.05 .{ }^{\dagger} P<0.01 .{ }^{\ddagger} P<0.001$. 

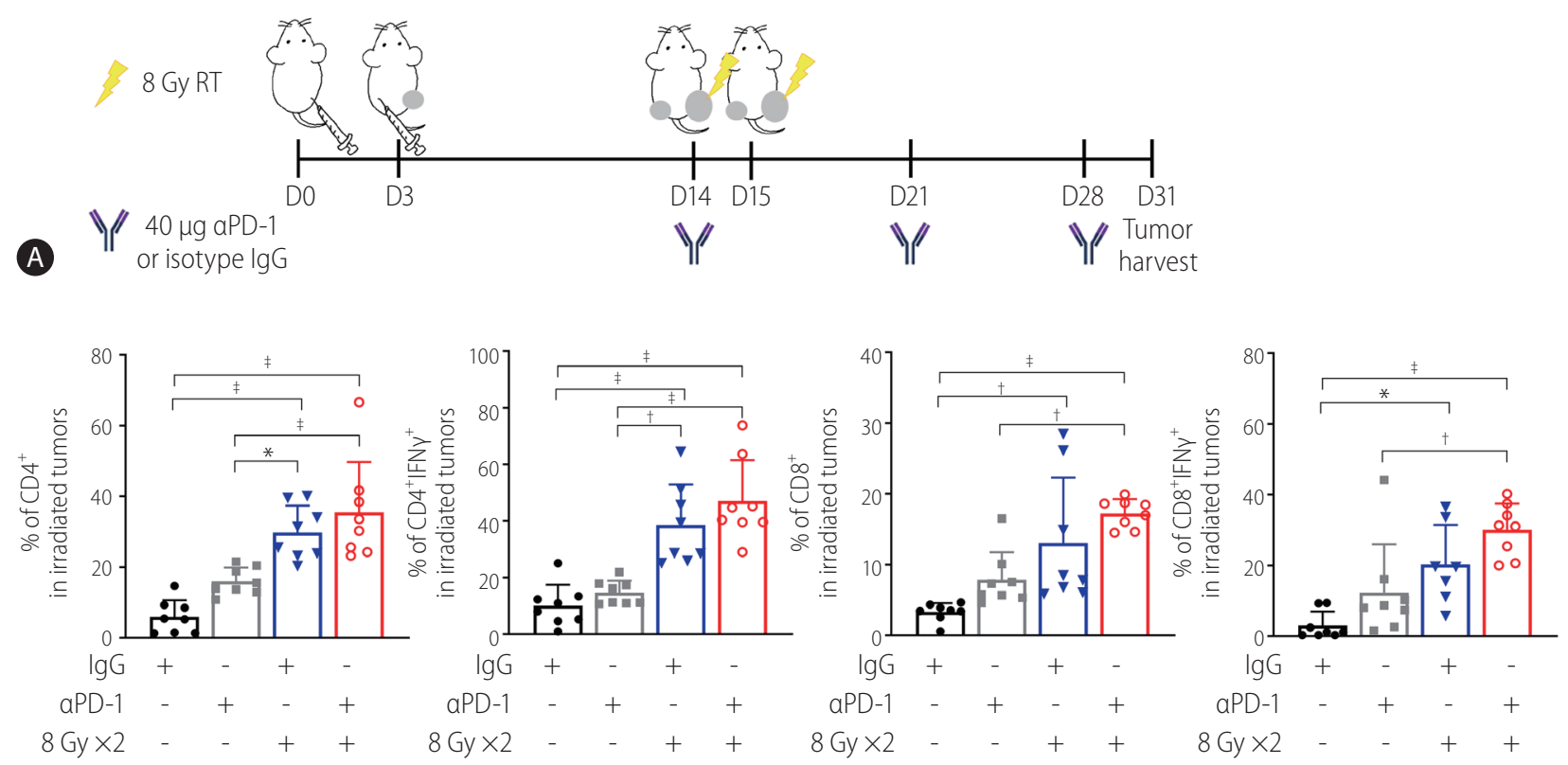

B
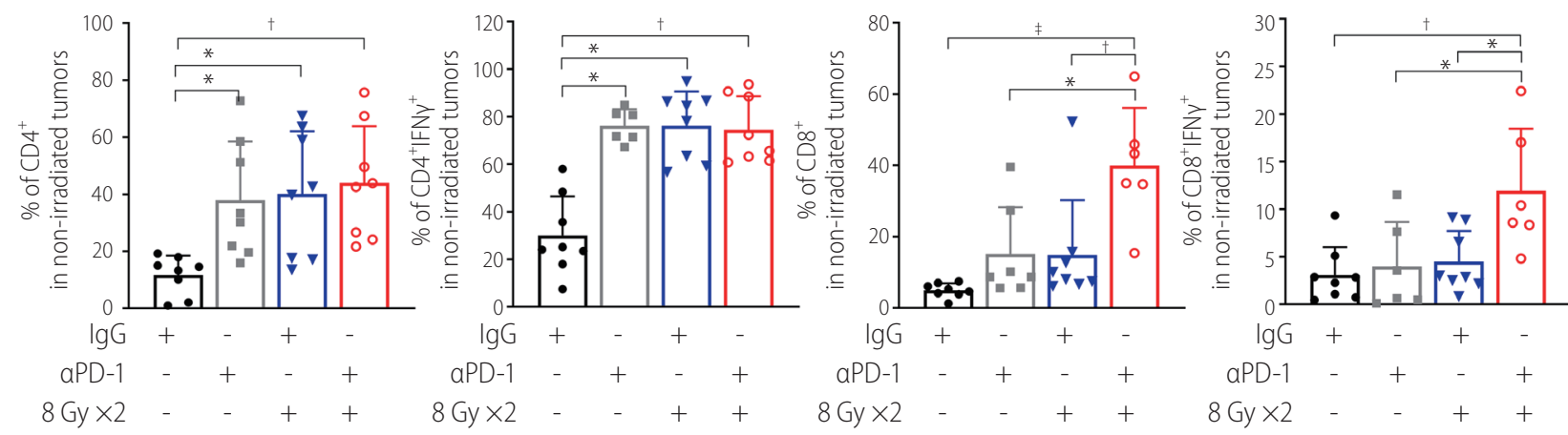

C

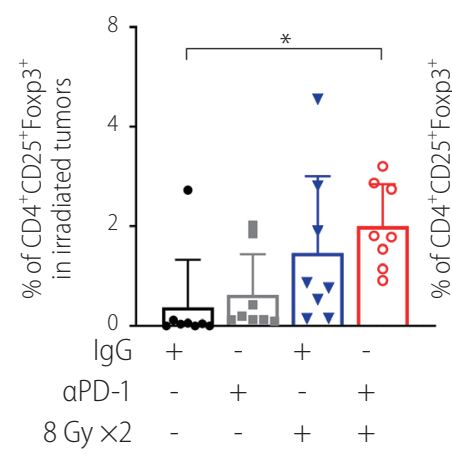

(D)

Figure 5. Anti-PD-1 boosts radiation-induced infiltration of activated T cells into both irradiated and non-irradiated tumors. (A) Scheme of anti-PD-1 and radiation treatment of a bilateral Hepa 1-6 tumor mouse model. (B) Flow cytometric analysis revealed that radiation but not anti-PD-1 alone increased infiltration of $C D 4^{+} T$ and $C D 8^{+} T$ cells and expression of IFN- $\gamma$ in these cells. This was further enhanced by combination with anti-PD-1. (C) Combination of radiation and anti-PD-1 increased population of activated CD4 ${ }^{+}$and CD8 ${ }^{+} T$ cells in non-irradiated tumors. Population of (D) T regulatory cells (Tregs) but not (E) CD11c dendritic cells (DCs) in irradiated and non-irradiated tumors was increased by cotreatment with anti-PD-1 and radiation. $\mathrm{RT}$, radiation therapy; $\mathrm{PD}-1$, programmed cell death 1 ; IgG, immunoglobulin $\mathrm{G}$; IFN, interferon. ${ }^{*} P<0.05 .{ }^{\dagger} P<0.01 .{ }^{\ddagger} P<0.001$. 
showed that in the irradiated tumors, 16 Gy administered in two fractions but not anti-PD-1 antibody treatment more significantly increased infiltration of $\mathrm{CD}^{+}$and $\mathrm{CD} 4^{+} \mathrm{IFN}-\gamma^{+} \mathrm{T}$ cells than the $\lg \mathrm{G}$ control did ( $P<0.001$, Fig. 5B). Similarly, 18 Gy administered in two fractions increased the infiltration of total $C D 8^{+}$and CD8 ${ }^{+}$IFN- $\gamma^{+} T$ cells (Fig. 5B). Cotreatment with anti-PD-1 antibodies and radiation further enhanced infiltration of $\mathrm{CD} 4^{+}$and $\mathrm{CD} 8^{+}$ $T$ cells more than treatment with the anti-PD1 antibodies alone but not radiation alone (Fig. 5B). Both radiation and the anti-PD-1 antibodies increased $C D 4^{+}$and $C D 4^{+} I F N-\gamma^{+} T$ cells but not $C D 8^{+} T$ cells in the non-irradiated tumors, while their cotreatment increased the total $C D 8^{+}$and $C D 8^{+} I F N \gamma^{+} T$ cells more than either treatment alone (Fig. 5C). Combination of radiation and the antiPD-1 antibodies increased enrichment of $\mathrm{CD}_{2} 5^{+} \mathrm{CD}^{+}{ }^{+}$Foxp $^{+}$Tregs in the tumors on both sides, compared to the IgG control treatment $(P<0.05$ and $P<0.01$, Fig. 5D), whereas no difference in $\mathrm{CD}_{11 c^{+}} \mathrm{DCs}$ was observed among the groups (Fig. 5E). Flow cytometric immunophenotyping revealed that the number of $\mathrm{CD}_{2} 2 \mathrm{O}^{+}$ $B$ cells, F4/80 ${ }^{+}$macrophages, Ly6G ${ }^{+} \mathrm{CD} 11 \mathrm{~b}^{+}$neutrophils, Ly6G ${ }^{-}$

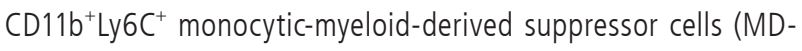
SCs), and Ly6G ${ }^{-}$CD11 $b^{+}$Ly6C ${ }^{\text {low }}$ granulocytic-MDSCs was not affected by monotherapy or co-therapy, except for that of $\mathrm{CD}^{+} \mathrm{T}$ cells (Supplementary Fig. 3).

\section{DISCUSSION}

Following the first published report on the abscopal effect, ${ }^{25}$ this phenomenon has been investigated in various cancers such as melanoma, renal cell carcinoma, and breast cancer both preclinically and clinically. However, compared with other malignancies, studies on the abscopal effect and its enhancement by immunotherapeutic agents in HCC is lacking, possibly because RT and immunotherapy have not been widely used for HCC management. The few studies that have used HCC models to evaluate the combined effect of RT and ICls described the effect of ICls on irradiated primary tumors but not the abscopal effect. ${ }^{20,21}$ Friedman et al. ${ }^{21}$ reported that in an orthotopic murine HCC model, PD-1 blockade enhanced the response to stereotactic radiation with 30 Gy in three fractions. Another study by Kim et al. ${ }^{20}$ demonstrated the synergistic effect of $10 \mathrm{~Gy}$ coadministered as a single fraction with an anti-PD-L1 antibody in reducing primary HCC tumor growth. Clinical observation of abscopal effects in HCC is extremely rare, ${ }^{26,27}$ and a recent study by Chen et al. ${ }^{28}$ showed that hypofractionated RT (up to $40 \mathrm{~Gy}$ ) inhibited non-irradiated tumor growth in a murine HCC model via suppression of MDSCs. To the best of our knowledge, this is the first study evaluating the enhancement of abscopal effects by a combination of radiation and ICls in a murine syngeneic HCC model.

A suggested mechanism of the abscopal effect is that it starts with the release of tumor antigens and danger-associated molecular patterns from tumor cells damaged by irradiation. ${ }^{19}$ The released tumor antigens are presented by antigen-presenting cells including DCs, which are drained into TDLNs. The drained DCs prime T cells, which subsequently become effector cells, migrate to the non-irradiated tumor cells, and induce adaptive immunity. ${ }^{29}$ In addition, radiation enhances the expression of type I IFN genes through cyclic guanosine monophosphate-adenosine monophosphate synthase (CGAS) and stimulator of IFN gene (STING) pathways, which are consequentially activated by the accumulation of cytosolic DNA. ${ }^{30}$ The increased IFN activates antigen-presenting cells and mediates the recruitment of effector $T$ cells to the target site. ${ }^{31}$ Our results showed a significant decrease in the growth of the non-irradiated tumors (Fig. 1C) and increases in the infiltration of activated $\mathrm{T}$ cells in the non-irradiated tumors and population of activated DCs in TDLNs (Figs. 2D, 3C) after irradiation with a total of $16 \mathrm{~Gy}$, which is in accordance with the proposed immunologic mechanism of the abscopal effect. Our study demonstrated that 16 Gy delivered in two fractions rather than 8 Gy in a single fraction showed a statistically significant difference in infiltration of activated $T$ cells compared with sham irradiation, implying the potential radiation dose-dependency of the abscopal effect. It is likely that higher doses may further effectively elevate levels of cytosolic DNA, triggering the CGAS/STING pathway ${ }^{32,33}$ However, radiation doses >12-18 Gy administered in a single fraction suppressed the abscopal effect by upregulating 3 ' repair exonuclease (TREX1) that degrades cytosolic DNA. ${ }^{30}$ Further investigation would be required to determine the optimal dosefraction required to maximize the abscopal effect.

The current study clearly showed that the anti-PD-1 antibodies enhanced the RT-induced abscopal effect (Fig. 4C) and the relevant immunologic phenomenon (Fig. $5 \mathrm{C}$ ) in murine HCC models. In particular, the infiltration of $\mathrm{CD} 8^{+} \mathrm{T}$ cells into the non-irradiated tumor was significantly higher when anti-PD-1 antibodies were co-administered with RT than when RT was administered alone. PD-1 is an immunoinhibitory receptor mainly expressed by mature T cells, B cells, and natural killer cells..$^{19}$ It specifically binds to PDL1 whose expression in tumor cells is mainly regulated by IFN- $\gamma$ and, thus, the interaction of PD-1 with PD-L1 results in T cell exhaustion. ${ }^{34}$ Blockade of PD-1/PD-L1 signaling restores effector T 
cell function to eradicate tumor cells. The antitumor activity of effector $T$ cells can potentially be enhanced by the immunogenic effect of radiation following their coadministration, which was demonstrated by our results.

In contrast to the antitumor immunity, 16 Gy radiation also induced expansion of immunosuppressive cells, including PD-L1-expressing DCs in TDLNs. DC PD-L1 suppresses the activation of $\mathrm{CD}^{+} \mathrm{T}$ cells via the PD-1/PD-L1 signaling axis. ${ }^{35,36}$ Thus, treatment with anti-PD-1 antibodies may allow DCs to reinvigorate T cells by blocking PD-1/PD-L1 interaction. Radiation also increased the infiltration of Tregs, another important immunosuppressive cell population, in both irradiated and non-irradiated tumors, which is consistent with the results of previous studies. ${ }^{37,38}$ An increased level of Tregs is associated with an unfavorable prognosis in various cancers including ovarian, breast, and gastric cancer and HCC. ${ }^{39-41}$ Furthermore, Tregs suppress cytotoxic T cell function with constitutive expression of CTLA-4, another immune checkpoint protein. Therefore, dual blockade of CTLA-4 and PD-1 may amplify the abscopal reaction triggered by RT in HCC.

Recent prospective clinical trials of PD-1 inhibitors such as nivolumab and pembrolizumab in HCC patients have reported overall ORRs of $17-20 \%$, leading to their approval by the US Food and Drug Administration as second-line treatment for patients who do not respond to sorafenib. 13,14 The ORRs with PD-1 inhibitors were higher than those obtained with the standard of care, sorafenib, but they are still unsatisfactory. Furthermore, the KEYNOTE-240 phase III trial, which investigated the benefit of pembrolizumab as a second-line therapy in patients with advanced HCC, failed to show the statistically significant superiority of pembrolizumab to the best supportive care with or without placebo in overall and progression-free survival. ${ }^{18}$ However, the overall survival of the pembrolizumab-treated group was satisfactory. ${ }^{18}$ Therefore, there is an urgent need to intensify the treatment efficacy, and the present study suggests that combining RT and ICls may potentially improve the unsatisfactory outcomes. Unfortunately, the relevant clinical data on RT and ICI cotreatment for advanced HCC is currently very limited. Recently, Yu et al. ${ }^{42}$ reported that previous or concurrent application of RT or both in the course of nivolumab treatment was related to prolonged progression-free or overall survival in advanced HCC patients. Despite the limitations of the previous study, including its retrospective design and small sample size, the results are meaningful and imply the clinical significance of the RT and immunotherapy combination. Currently, there are several ongoing clinical trials investigating combinations of various ICls and RT techniques including external beam RT and selective internal RT for advanced HCC. ${ }^{19}$ However, trials focusing on metastatic HCC are still limited. Our findings may be extended to the design of a clinical study to evaluate the improvement of systemic disease control by mutual intensification of immunologic activation, which could contribute to developing strategies for overcoming resistance to PD-1 inhibitors in HCC.

There are some limitations in the present study. This study used a single murine HCC Hepa 1-6 cell line and, therefore, further validation is required in different HCC models. In addition, other factors associated with the tumor microenvironment, such as intrinsic tumor factors, could also be associated with the abscopal effect. There was a discrepancy between abscopal effects on the non-irradiated tumors induced by 16 Gy irradiation in addition to the size of the sham control tumors as shown in Figures 1 and 4 . We speculate that the size of non-irradiated tumors at the time of irradiation may affect the abscopal effect, which needs further investigation. Although the effectiveness of cotreatment in boosting the abscopal phenomenon was established in the present study, further studies with meticulous controls would be necessary.

In conclusion, using a syngeneic murine bilateral HCC model and flow cytometric profiling of intratumor immune cells, we showed that delivering 16 Gy in two fractions induced an abscopal effect, which was boosted by cotreatment with anti-PD-1 antibodies. Based on our preclinical data, future clinical trials investigating the effect of RT and ICls in the metastatic setting are warranted.

\section{Authors' contribution}

WG Ahn and SY Kim performed the experiments. GS Yoo, W Kang and $\mathrm{C}$ Choi analyzed the data and prepared the manuscript. C Choi and HC Park designed and supervised the study. GS Yoo, C Choi and HC Park wrote the manuscript. All authors read and approved the final manuscript for publication.

\section{Acknowledgements}

This work was supported by a National Research Foundation of Korea (NRF) grant funded by the Korea government (MSIT) (NRF2018R1A2B2002835).

\section{Conflicts of Interest}

The authors have no conflicts to disclose. 


\section{SUPPLEMENTARY MATERIAL}

Supplementary material is available at Clinical and Molecular Hepatology website (http://www.e-cmh.org).

\section{REFERENCES}

1. Siva S, MacManus MP, Martin RF, Martin OA. Abscopal effects of radiation therapy: a clinical review for the radiobiologist. Cancer Lett 2015;356:82-90.

2. Demaria $S, N g$ B, Devitt ML, Babb JS, Kawashima N, Liebes $L$, et al. Ionizing radiation inhibition of distant untreated tumors (abscopal effect) is immune mediated. Int J Radiat Oncol Biol Phys 2004;58:862-870.

3. Reynders K, Illidge T, Siva S, Chang JY, De Ruysscher D. The abscopal effect of local radiotherapy: using immunotherapy to make a rare event clinically relevant. Cancer Treat Rev 2015;41:503-510.

4. Elsegood CL, Tirnitz-Parker JE, Olynyk JK, Yeoh GC. Immune checkpoint inhibition: prospects for prevention and therapy of hepatocellular carcinoma. Clin Transl Immunology 2017;6:e161.

5. Ngwa W, Irabor OC, Schoenfeld JD, Hesser J, Demaria S, Formenti SC. Using immunotherapy to boost the abscopal effect. Nat Rev Cancer 2018;18:313-322.

6. Wang SJ, Haffty B. Radiotherapy as a new player in immuno-oncology. Cancers (Basel) 2018;10:515.

7. Sharabi AB, Lim M, DeWeese TL, Drake CG. Radiation and checkpoint blockade immunotherapy: radiosensitisation and potential mechanisms of synergy. Lancet Oncol 2015;16:e498-e509.

8. Kang J, Demaria S, Formenti S. Current clinical trials testing the combination of immunotherapy with radiotherapy. J Immunother Cancer 2016:4:51.

9. Ghouri YA, Mian I, Rowe JH. Review of hepatocellular carcinoma: epidemiology, etiology, and carcinogenesis. J Carcinog 2017;16:1.

10. Lafaro KJ, Demirjian AN, Pawlik TM. Epidemiology of hepatocellular carcinoma. Surg Oncol Clin N Am 2015;24:1-17.

11. Bruix J, Raoul JL, Sherman M, Mazzaferro V, Bolondi L, Craxi A, et al. Efficacy and safety of sorafenib in patients with advanced hepatocellular carcinoma: subanalyses of a phase III trial. J Hepatol 2012;57:821-829.

12. Yu JI, Park HC. Radiotherapy as valid modality for hepatocellular carcinoma with portal vein tumor thrombosis. World J Gastroenterol 2016;22:6851-6863.

13. El-Khoueiry AB, Sangro B, Yau T, Crocenzi TS, Kudo M, Hsu C, et al. Nivolumab in patients with advanced hepatocellular carcinoma (CheckMate 040): an open-label, non-comparative, phase 1/2 dose escalation and expansion trial. Lancet 2017;389:2492-2502.

14. Zhu AX, Finn RS, Edeline J, Cattan S, Ogasawara S, Palmer D, et al. Pembrolizumab in patients with advanced hepatocellular carcinoma previously treated with sorafenib (KEYNOTE-224): a nonrandomised, open-label phase 2 trial. Lancet Oncol 2018;19:940952.

15. Kelley RK, Abou-Alfa GK, Bendell JC, Kim TY, Borad MJ, Yong WP, et al. Phase I/II study of durvalumab and tremelimumab in patients with unresectable hepatocellular carcinoma (HCC): phase I safety and efficacy analyses. J Clin Oncol 2017;35:4073.

16. Abou-Alfa GK, Chan SL, Furuse J, Galle PR, Kelley RK, Qin S, et al. A randomized, multicenter phase 3 study of durvalumab (D) and tremelimumab $(\mathrm{T})$ as first-line treatment in patients with unresectable hepatocellular carcinoma (HCC): HIMALAYA study. J Clin Oncol 2018:36:TPS4144.

17. Yoo GS, Yu JI, Park HC. Proton therapy for hepatocellular carcinoma: current knowledges and future perspectives. World J Gastroenterol 2018;24:3090-3100.

18. Finn RS, Ryoo BY, Merle P, Kudo M, Bouattour M, Lim HY, et al. Pembrolizumab as second-line therapy in patients with advanced hepatocellular carcinoma in KEYNOTE-240: a randomized, doubleblind, phase III trial. J Clin Oncol 2020;38:193-202.

19. Choi C, Yoo GS, Cho WK, Park HC. Optimizing radiotherapy with immune checkpoint blockade in hepatocellular carcinoma. World J Gastroenterol 2019;25:2416-2429.

20. Kim KJ, Kim JH, Lee SJ, Lee EJ, Shin EC, Seong J. Radiation improves antitumor effect of immune checkpoint inhibitor in murine hepatocellular carcinoma model. Oncotarget 2017;8:41242-41255.

21. Friedman D, Baird JR, Young KH, Cottam B, Crittenden MR, Friedman $S$, et al. Programmed cell death-1 blockade enhances response to stereotactic radiation in an orthotopic murine model of hepatocellular carcinoma. Hepatol Res 2017;47:702-714.

22. Wu CJ, Tsai YT, Lee IJ, Wu PY, Lu LS, Tsao WS, et al. Combination of radiation and interleukin 12 eradicates large orthotopic hepatocellular carcinoma through immunomodulation of tumor microenvironment. Oncoimmunology 2018;7:e1477459.

23. Yu JI, Choi C, Shin SW, Son A, Lee GH, Kim SY, et al. Valproic acid sensitizes hepatocellular carcinoma cells to proton therapy by suppressing NRF2 activation. Sci Rep 2017;7:14986.

24. Shin SW, Jung W, Choi C, Kim SY, Son A, Kim H, et al. Fucoidanmanganese dioxide nanoparticles potentiate radiation therapy by co-targeting tumor hypoxia and angiogenesis. Mar Drugs 2018;16:510.

25. Mole RH. Whole body irradiation; radiobiology or medicine? $\mathrm{Br} J$ Radiol 1953;26:234-241.

26. Ohba K, Omagari K, Nakamura T, Ikuno N, Saeki S, Matsuo I, et al. Abscopal regression of hepatocellular carcinoma after radiotherapy for bone metastasis. Gut 1998:43:575-577.

27. Okuma K, Yamashita H, Niibe Y, Hayakawa K, Nakagawa K. Abscopal effect of radiation on lung metastases of hepatocellular carci- 


\section{CLINICAL and MOLECULAR
HEPATOLOGY}

noma: a case report. J Med Case Rep 2011;5:111.

28. Chen J, Wang Z, Ding Y, Huang F, Huang W, Lan R, et al. Hypofractionated irradiation suppressed the off-target mouse hepatocarcinoma growth by inhibiting myeloid-derived suppressor cell-mediated immune suppression. Front Oncol 2020;10:4.

29. Liu Y, Dong Y, Kong L, Shi F, Zhu H, Yu J. Abscopal effect of radiotherapy combined with immune checkpoint inhibitors. J Hematol Oncol 2018;11:104.

30. Vanpouille-Box C, Alard A, Aryankalayil MJ, Sarfraz Y, Diamond JM, Schneider RJ, et al. DNA exonuclease Trex1 regulates radiotherapyinduced tumour immunogenicity. Nat Commun 2017;8:15618.

31. Ivashkiv LB. IFNY: signalling, epigenetics and roles in immunity, metabolism, disease and cancer immunotherapy. Nat Rev Immunol 2018:18:545-558.

32. Osipov A, Arkhangelskaya E, Vinokurov A, Smetanina N, Zhavoronkov A, Klokov D. DNA comet Giemsa staining for conventional bright-field microscopy. Int J Mol Sci 2014;15:6086-6095.

33. Durante M, Formenti SC. Radiation-induced chromosomal aberrations and immunotherapy: micronuclei, cytosolic DNA, and interferon-production pathway. Front Oncol 2018;8:192.

34. Im SJ, Ha SJ. Re-defining T-cell exhaustion: subset, function, and regulation. Immune Netw 2020;20:e2.

35. Dovedi SJ, Lipowska-Bhalla G, Beers SA, Cheadle EJ, Mu L, Glennie $M J$, et al. Antitumor efficacy of radiation plus immunotherapy depends upon dendritic cell activation of effector CD8+ T cells. Cancer
Immunol Res 2016;4:621-630.

36. Versteven M, Van den Bergh JMJ, Marcq E, Smits ELJ, Van Tendeloo VFI, Hobo W, et al. Dendritic cells and programmed death-1 blockade: a joint venture to combat cancer. Front Immunol 2018;9:394.

37. Muroyama Y, Nirschl TR, Kochel CM, Lopez-Bujanda Z, Theodros D, Mao $W$, et al. Stereotactic radiotherapy increases functionally suppressive regulatory T cells in the tumor microenvironment. Cancer Immunol Res 2017;5:992-1004.

38. Kachikwu EL, Iwamoto KS, Liao YP, DeMarco JJ, Agazaryan N, Economou JS, et al. Radiation enhances regulatory $T$ cell representation. Int J Radiat Oncol Biol Phys 2011;81:1128-1135.

39. Curiel TJ, Coukos G, Zou L, Alvarez X, Cheng P, Mottram P, et al. Specific recruitment of regulatory $T$ cells in ovarian carcinoma fosters immune privilege and predicts reduced survival. Nat Med 2004;10:942-949.

40. Sage PT, Schildberg FA, Sobel RA, Kuchroo VK, Freeman GJ, Sharpe AH. Dendritic cell PD-L1 limits autoimmunity and follicular T cell differentiation and function. J Immunol 2018;200:2592-2602.

41. Tu JF, Ding YH, Ying XH, Wu FZ, Zhou XM, Zhang DK, et al. Regulatory $T$ cells, especially ICOS+ FOXP3+ regulatory $T$ cells, are increased in the hepatocellular carcinoma microenvironment and predict reduced survival. Sci Rep 2016;6:35056.

42. Yu JI, Lee SJ, Lee J, Lim HY, Paik SW, Yoo GS, et al. Clinical significance of radiotherapy before and/or during nivolumab treatment in hepatocellular carcinoma. Cancer Med 2019;8:6986-6994. 